\title{
Measurement of third-order elastic constants and stress dependent coefficients for steels
}

\author{
Sennosuke Takahashi (D)
}

\begin{abstract}
Background: There has been little discussion of the third-order elastic constants of steels in the literature until now. In this study, the precise second- and third-order elastic constants of polycrystalline steels were measured under adiabatic and isothermal conditions.

Method: To measure the minute change in the propagation time of the elastic wave corresponding to the tensile stress, the uniform and isotropic specimens were processed with high precision, the measuring instruments were strictly calibrated, and the temperature of the measurement chamber was kept constant. The author proposes an experimental formula to obtain the third-order elastic constants of steels. The stress dependent coefficients $\alpha_{i j}$ in this formula are absolutely necessary to obtain the third-order elastic constants.
\end{abstract}

Results: The obtained stress dependent coefficients clearly indicated that there is a special relationship between the directions of stress and that of the oscillation of the elastic wave. When the frequency direction of the elastic wave matched the direction of the applied stress, $\alpha_{i j}$ became a larger negative value. Lamè constants and Murnaghan's third-order elastic constants $\ell, m, n$ were obtained for four types of steels.

Conclusions: The second- and third-order elastic constants under adiabatic conditions were smaller than those under isothermal conditions. Oscillation of crystal lattice is nonlinear and this is observed as the third-order elastic constants. Therefore, it is possible to obtain the knowledge on the internal stress and the thermal properties of the materials. This is also the basis of theoretical discussion of the thermal expansion coefficients.

Keywords: Second-and third-order elastic modulus, Elastic wave, Stress dependent coefficient, Polycrystalline material

\section{Introduction}

The first study of the theory and measurement of the third-order elastic constants of practical materials was published by Hughes and Kelly (1953). D.Lazarus reported the third-order elastic constants of the single crystals like $\mathrm{KCl}, \mathrm{Cu}$ etc by measureing propagation velocity of elastic wave under hydrostatic pressure (Lazarus 1949) and it may useful in comparison of the theories of finite strain proposed by Murnagham (1951). R.N.Thurston published a paper on the theoretical analysis of the propagation of elastic wave (Thurston and Brugger 1964). D.M. Egle et al. carried out the measurement of the third-order elastic

Correspondence: fwnk4784@nifty.com

National Research Institute for Metals, 6-13-12 Kanamori, Machida, Tokyo 194-0012, Japan constants for rail steel using Hughes's result (Egle and Bray 1976). S.Takahashi got the U.S.patent on the stress measurement and its equipment according to the method of the present paper (Takahashi 2007). T.Batemen et al. reported that the third-order elastic constant of semiconductor was related to thermal expansion coefficient and Güneisen constants (Bateman et al. 1961). As described above, the knowledge on the third-order elastic constants contributes to the study of physical properties of various materials.

This paper describes the measurement of the thirdorder elastic constants of four common steels. The stress applied to the specimen was increased stepwise, and the velocity of the elastic wave was measured at every step using a high accuracy measuring technique. The shape 
and dimensions of the specimen were controlled as precisely as possible and the measuring equipment was also accurately calibrated. The change in room temperature was kept to $1^{\circ} \mathrm{C}$ or less during the measurement. Secondand third-order elastic constants contribute to the change in the propagation velocity of the elastic wave caused by stress (Hughes and Kelly 1953; Takahashi and Motegi 2015).

The author proposed a simple equation of the propagation velocity under stress by introducing the coefficient $\alpha_{i j}$ including the second- and third-order elastic constants. The value of $\alpha_{i j}$ can be obtained from the measured stress and the change ratio in propagation velocity. The coefficients $\alpha_{i j}$ is absolutely necessary to obtain the third-order elastic constants. When the frequency direction of the elastic wave matched the direction of the applied stress, the value of $\alpha_{i j}$ became more negative. This means that the coincidence of the frequency direction with the applied stress greatly contributed to the propagation velocity of the wave. The value of $\alpha_{i j}$ is based on the stress-strain relation. Therefore it is necessary to obtain the vales of $\alpha_{i j}$ on this relationship.

\section{Experimental method}

\section{Test specimen}

Figure 1 shows the dimensions and coordinates of the test specimens. The specimens were designed to be attached to the tensile testing machine and make it easy to measure their elastic waves. Coordinates numbered 1, 2, and 3 were used instead of $x, y$, and $z$. The long axis direction of the specimen was denoted 1 , and the directions perpendicular to it were denoted 2 and 3 . The propagation velocity of the longitudinal wave in direction 1 was expressed as $V_{11}$, while that of the transvers wave to direction 1 and vibration in direction 2 was expressed as $V_{12}$. For the propagation velocity $\mathrm{V}$, the same subscripts express the longitudinal wave, while different subscripts express the transverse wave. $T_{11}$ represents the tensile stress in the direction of the long axis of the specimen. Table 1 lists the chemical compositions of the S20C(AISI 1020), S30C(AISI 1030), S40C(AISI 1039) and S50C(AISI 1049) test specimens.

\section{Stress dependent coefficients of elastic wave $\alpha_{i j}$}

The propagation velocity $V_{i j}$ of the elastic wave in the specimen under an applied stress of $T_{11}$ is expressed as

$$
V_{i j}=V_{0}\left(1+\alpha_{i j} \frac{T_{11}}{E}\right)
$$

where $V_{0}$ is the propagation velocity of the elastic wave under non-loaded state, $\alpha_{i j}$ is the stress dependent coefficient of the elastic wave, and $E$ is Young's modulus. The expression of $V_{i j}$ by Hughes and Kelly (1953) and the authors Takahashi and Motegi (2015) is

$\rho_{0} V_{11}^{2}=\lambda+2 \mu+\frac{T_{11}}{E}[5 \lambda+10 \mu+2 \ell+4 m-2 v(\lambda+2 \ell)]$

where $\lambda, \mu$ are Lamè constants, $\ell, m, n$ are the Murnaghan's third order elastic constants, $v$ is Poisson's ratio and $\rho_{0}$ is the density in the non-deformed state. The formula (1) is based on an equation previously introduced by the authors Takahashi and Motegi (2015) and it can be rewritten as

$$
\begin{aligned}
V_{11}^{2} & =V_{0}^{2}\left(1+\alpha_{11} \frac{T_{11}}{E}\right)^{2} \approx V_{0}^{2}\left(1+2 \alpha_{11} \frac{T_{11}}{E}\right) \\
V_{0}^{2} & =\frac{\lambda+2 \mu}{\rho_{0}}
\end{aligned}
$$

From the relations described above, $\alpha_{11}$ is given as follows,

$$
\alpha_{11}=\frac{1}{2(\lambda+2 \mu)}[5 \lambda+10 \mu+2 \ell+4 m-2 v(\lambda+2 \ell)]
$$
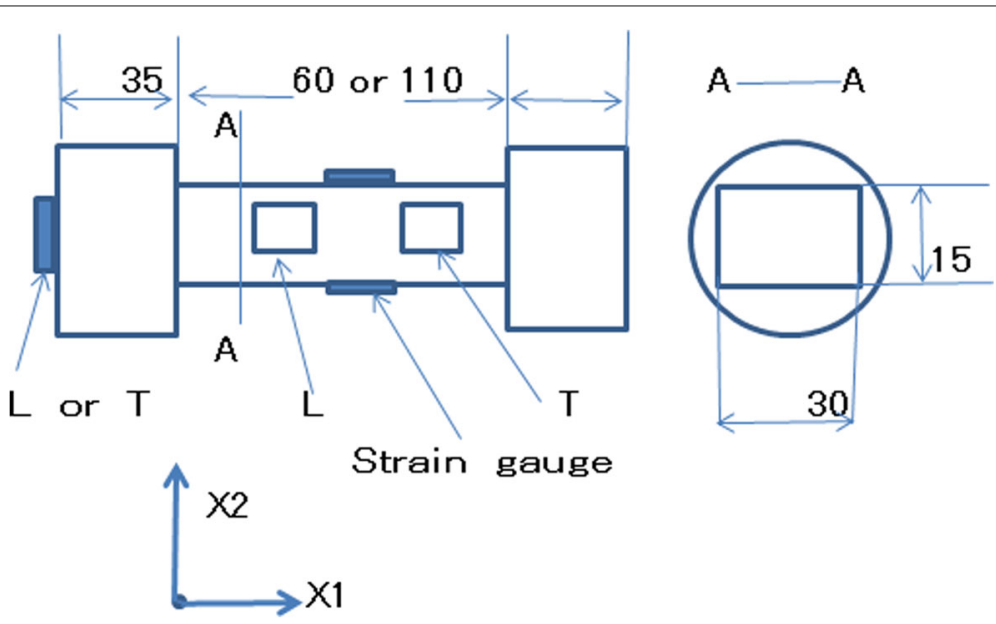

Fig. 1 Schematic diagram of specimen $L$ and $T$ : longitudinal and transvers transducers 
Table 1 Chemical composition of S20C to S50C specimens(wt\%)

\begin{tabular}{lllll}
\hline Sample & $\mathrm{C}$ & $\mathrm{Si}$ & $\mathrm{Mn}$ & $\mathrm{Ni}+\mathrm{Cr}$ \\
\hline S20C & 0.22 & 0.29 & 0.52 & \\
S30C & 0.32 & 0.31 & 0.81 & \\
S40C & 0.40 & 0.28 & 0.78 & 0.12 \\
S50C & 0.49 & 0.31 & 0.83 & 0.12 \\
\hline
\end{tabular}

In the same way, the formulae of other $\alpha_{22}, \alpha_{21}, \alpha_{12}$ and $\alpha_{23}$ can be obtained.

For example,

$$
\alpha_{22}=\frac{1}{2(\lambda+2 \mu)}[\lambda+2 \ell-v(6 \lambda+10 \mu+4 \ell+4 m)]
$$

The coefficient $\alpha_{i j}$ is used to obtain Murnaghan's thirdorder elastic constants $\ell, m, n$ as follows.

$$
\begin{aligned}
\ell & =\frac{\left(2 \alpha_{11}-5\right)(\lambda+2 \mu)}{2(1-2 v)}-\frac{2 m-v \lambda}{1-2 v} \\
m & =\left[\frac{\alpha_{11}-\alpha_{22}}{2(1+v)}-1\right](\lambda+2 \mu)-\frac{\mu}{2} \\
n_{12} & =\frac{2}{v}\left[-(a+4 v)+2 v(a+\mu)+2 \mu \alpha_{12}\right] \\
n_{21} & =\frac{2}{v}\left[-(a+2 \mu)+2 v(a+2 \mu)+2 \mu \alpha_{21}\right] \\
n_{23} & =2\left[a-2 v(a+3 \mu)-2 \mu \alpha_{23}\right]
\end{aligned}
$$

here $a=\lambda+m$

\section{Measurement of the propagation velocity of elastic wave in the applied stress direction}

The stress in the gripping regions is complicated and should be eliminated, so two kinds of specimens with identical grip sizes but different gauge length were prepared. Hereafter the symbol a denotes the long specimen while $b$ denotes the short specimen. The propagation times under stress free are written as

$$
t_{0 a}=L_{a} / V_{0}, t_{0 b}=L_{b} / V_{0},
$$

where $L_{a}, L_{b}$ are the total length of the specimens under stress free conditions, and $t_{a}, t_{b}$ are the propagatioon time under applied stress.

The differences in propagation time are written as $\Delta t_{a}=t_{a}-t_{0 a}$ and $\Delta t_{b}=t_{b}-t_{0 b}$.

The propagation time in the applied stress direction is obtained from formula (1) as follows,

$$
t=\frac{L_{g}}{V_{g}}+\frac{L_{m}\left(1+\frac{T_{11}}{E}\right)}{V_{0}\left(1+\alpha_{11} \frac{T_{11}}{E}\right)}
$$

where $L_{m}$ is the length of the gauge part in the non-loaded state, $L_{g}$ is the grip length under the applied stress, $V_{g}$ is the average velocity of the wave passing through grip part. In the case of a longitudinal wave propagating in the applied stress direction of the long specimen, $\Delta t_{a} / t_{0 a}$ can be written using an approximate calculation as

$$
\frac{\Delta t_{a}}{t_{0 a}}=\frac{\left(\frac{L_{g}}{V_{g}}-\frac{L_{m}}{V_{0}}\right)}{\frac{L_{a}}{V_{0}}}+\frac{L_{m a}}{L_{a}}\left(1-\alpha_{11}\right) \frac{T_{11}}{E}
$$

$\Delta t_{a} / t_{0 a}$ and $\Delta t_{b} / t_{0 b}$ can be obtained by measuring the time of the propagating elastic wave.

In a similar manner, for the short specimen,

$$
\frac{\Delta t_{b}}{t_{0 b}}=\frac{\left(\frac{L_{g}}{V_{g}}-\frac{L_{m}}{V_{0}}\right)}{\frac{L_{b}}{V_{0}}}+\frac{L_{m b}}{L_{b}}\left(1-\alpha_{11}\right) \frac{T_{11}}{E}
$$

The grip and the gauge parts are expressed separately in the above formulae. Using above two formulae, $\alpha_{11}$ is given as

$$
\begin{aligned}
& \alpha_{11}=1-\frac{E}{T_{11}}\left[\frac{L_{a}}{\Delta L} \cdot\left(\frac{\Delta t_{a}}{t_{0 a}}\right)-\frac{L_{b}}{\Delta L} \cdot\left(\frac{\Delta t_{b}}{t_{0 b}}\right)\right] \\
& \Delta L=L_{a}-L_{b}=50
\end{aligned}
$$

The transvers wave of $\alpha_{12}$ is also obtained from applying the measured $\Delta t_{a} / t_{0 a}$ and $\Delta t_{b} / t_{0 b}$ data to the formula (9).

\section{Measurement of propagation velocity of elastic wave in direction orthogonal to the tensile stress axis}

The propagation time of the elastic wave $t$ measured at the side of the specimen is defined by the following,

$$
t=\frac{W\left(1-\frac{\nu T_{11}}{E}\right)}{V_{0}\left(1+\frac{\alpha_{i j} T_{11}}{E}\right)}
$$

where $\mathrm{W}$ is width of the non-loaded specimen, and $v$ is Poisson's ratio. From the approximate calculation of formula(10), the coefficient related to the elastic wave in the direction of $2 j$ is given as

$$
\alpha_{2 j}=-v-\frac{E}{T_{11}}\left(\frac{\Delta t}{t_{0}}\right)
$$

The coefficient $\alpha_{22}$ for longitudinal waves and $\alpha_{21}, \alpha_{23}$ for transverse waves can be obtained from formula (11) using the respective value of $\left(\frac{\Delta t}{t_{0}}\right)$ from the measurements of S20C to S50C specimens.

\section{Measurement equipment}

An Instron type tensile testing machine and a computerized strain measurement apparatus were used in this work. The load cell was compensated using the standard gauge. The strain of the specimen was measured by strain gauge adhered on both sides of the gauge region as shown in Fig. 1. Measurement of the propagation time of the elastic wave was performed with a device having a time resolution of 10ns as shown in Fig. 2 (Takahashi and Motegi 1987). The grip holder of the tensile testing machine was designed and manufactured to pull the 


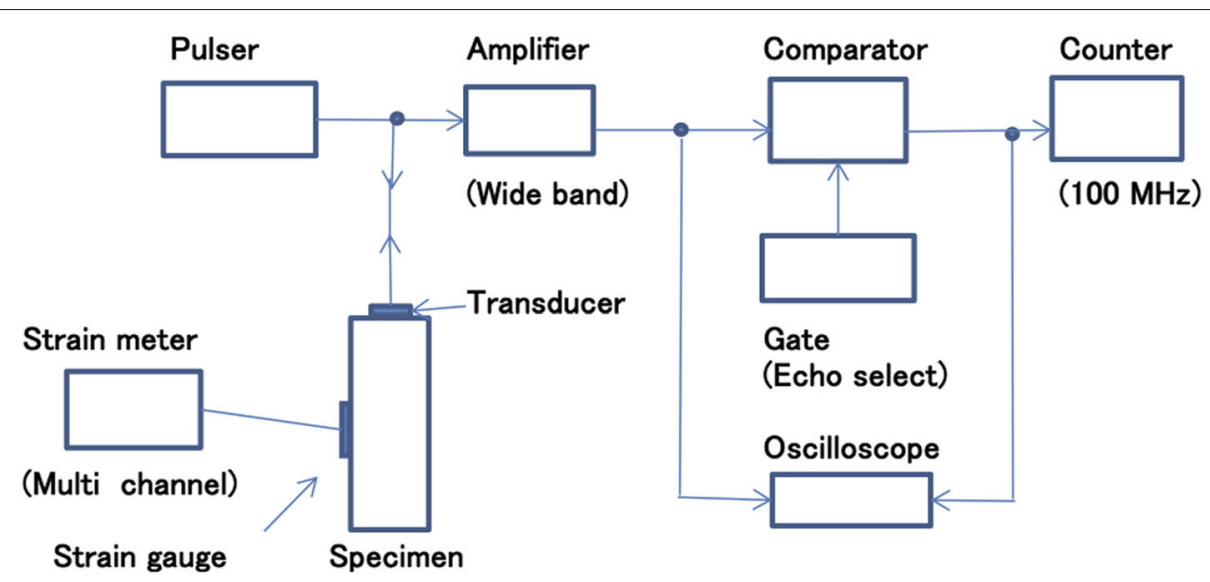

Fig. 2 Schematic diagram of the measurement system

leading wire of transducer from the both ends of the specimen (Takahashi and Takahashi 2007). PZT type(2-5MHz) piezoelectric resonators, plates of $10 \times 10 \mathrm{~mm}^{2}$ in size, were used as transducers for the longitudinal and tansvers waves.

\section{Results}

The stress applied to the specimen was increased in steps of 5.4MPa. The stress, strain, Poisson's ratio, propagation time of longitudinal and transvers waves were measured after each increase. Figure 3 shows the stress-strain curve for S30C sample. Figure 4 shows the relationship between the ratio of change in the propagation time with stress for S30C, obtained by measuring the longitudinal and transvers waves propagating parallel and perpendicular to

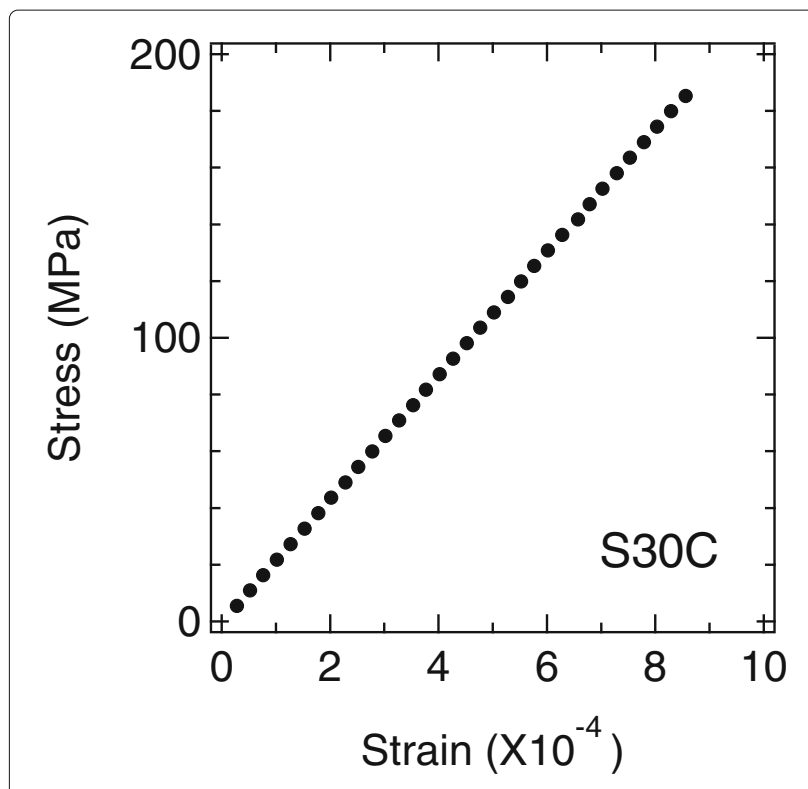

Fig. 3 Stress vs strain for $\mathrm{S30C}$ specimen the stress axis. The coefficient $\alpha_{i j}$ was obtained by measurering the gradient of the stress vs change ratio of the propagation time curve shown in Fig. 4 to formula (9) and (11) for long and short specimens.

The values of Lamè constant, Young's modulus, Poisson's ratio, third-order elastic constants and $\alpha_{i j}$ obtained by the stress-strain curve of tencile testing machine were set as values under isothermal conditions. On the other hand, these obtained from the measured values of the propagating time of the elastic wave were taken as values of adibatic conditions. The measured values of $\alpha_{11}$,

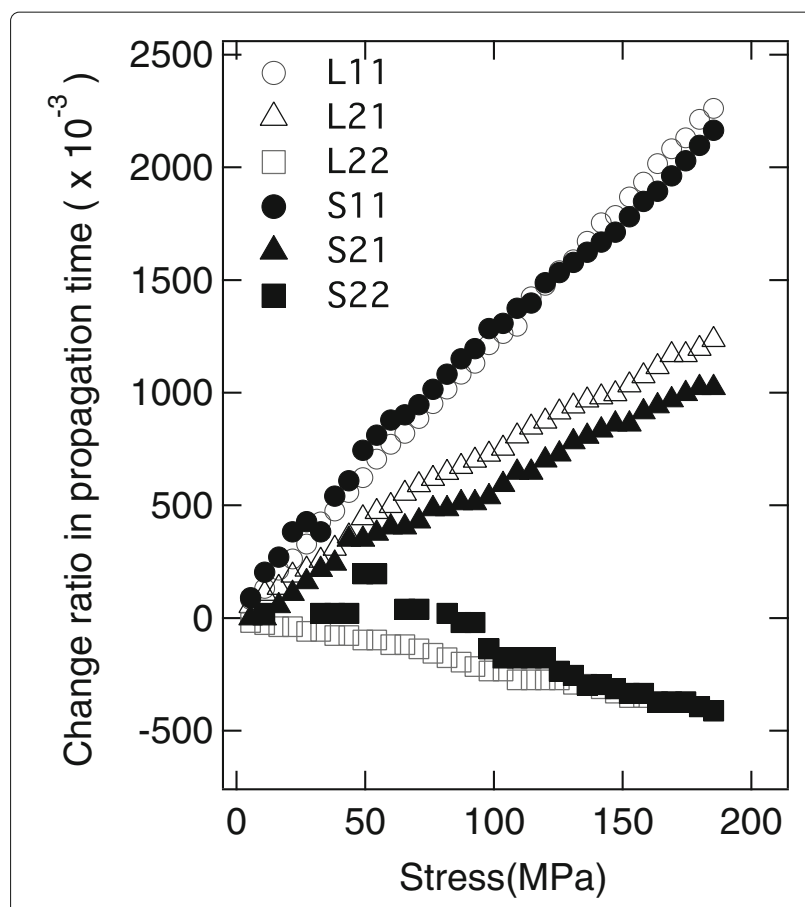

Fig. 4 Ratio of change in propagation velocity of elastic wave vs stress for S30C L:long specimen, S:short specimen 
$\alpha_{12}, \alpha_{21}, \alpha_{22}$ and $\alpha_{23}$ in the isothermal and adiabatic states are shown in Table 2. These were different owing to the different elastic constants measured by the tensile test or from the adiabatic elastic wave. The second-order elastic constants of the isothermal and adiabatic measurements are shown in Table 2 alongside the third-order elastic constants.

Figure 5 shows the stress dependent coefficients $\alpha_{11}$, $\alpha_{12}, \alpha_{21}, \alpha_{22}$ and $\alpha_{23}$ of each specimen. It was clear that $\alpha$ having coordinates 11 or 21 showed a larger negative value. It is considered that the agreement between the stress and oscillation direction of the elastic wave greatly displaced $\alpha_{i j}$ towards a more negative value. The measured values of the third-order elastic constants of each specimen in adiabatic state are shown in Fig. 6.

\section{Discussion}

The main goal of this study was to accurately measure the change in the propagation time of elastic waves in the material with stress and obtain a mathematical formula connecting theory and experiments to derive the third-order elastic constants. An empirical formula consisting of the stress dependent coefficient $\alpha_{i j}$ related with the third-order elastic constants was obtained based on the mathematical formula derived by Hughes and Kelly (1953) and the present author
Takahashi and Motegi (2015). Care was taken in preparing the specimens to precise dimensions, using wellcalibrated measument equipment, and maintaining good temperature control during the measurement to obtain precise $\alpha_{i j}$ data.

The basis of this experiment is to measure the value of $\alpha_{i j}$. The value of $\alpha_{i j}$ can be obtained from the gradient of changing ratio of elastic wave propagation time to the applied stress. Therefore from this viewpoint, $\alpha_{i j}$ should be determined. When the direction of the stress matches the direction of the vibration wave, $\alpha_{i j}$ has a larger negative value compared with in the other cases. It means the decrease of propagation velocity of the elastic wave. Thus, this coefficient $\alpha_{i j}$ also has a relationship to the propagation velocity of the elastic waves. Murnaghan's third order elastic constants could be calculated using the obtained coefficients $\alpha_{i j}$. As described above, of the obtained thirdorder elastic constants, $\ell, m$ and $n$ were negative for all specimens.

Table 2(B)(C) show the differences between isothermal and adiabatic elastic constants. The difference in the third-order elastic constants are larger than the difference in the second-order ones. O.M.Krasinikov reported no significant difference between isothermal and adiabatic elastic constants (Krasilnikov 1977). However, there is a relatively large difference between $m$ and $n$ in isothermal state and adiabatic state.

Table 2 Coefficient $\alpha_{i j}$ and second- and third-order elastic constants in the isothermal and adiabatic states

(A) Stress dependent coefficients of $\boldsymbol{\alpha}_{i j}$

$\begin{array}{lll}\alpha_{i j} & \text { S20iso } & \text { S20adi } \\ \alpha_{11} & -1.484 & -1.450 \\ \alpha_{22} & 0.232 & 0.227 \\ \alpha_{12} & 0.282 & 0.291 \\ \alpha_{21} & -1.209 & -1.197 \\ \alpha_{23} & 0.408 & 0.399\end{array}$

S30iso
-1.620
0.161
0.157
-1.443
0.116

(B) Lamè constants and Young's modulus $\left(\times 10^{3} \mathrm{MPa}\right)$

$\begin{array}{llll}\lambda & 115 & 114 & 98 \\ \mu & 83 & 82 & 86 \\ \mathrm{E} & 214 & 211 & 217\end{array}$

(C) Murnaghan's third order elastic constants $\left(\times 10^{3} \mathrm{MPa}\right)$

$\begin{array}{lll}\ell & -940 & -932 \\ m & -346 & -335 \\ n_{12} & -1161 & -1025 \\ n_{21} & -1192 & -1187 \\ n_{23} & -619 & -601 \\ \rho & 7.833 & \\ v & 0.291 & 0.290\end{array}$

$\rho$ : density, $v$ : Poisson's ratio
$-797$

$-355$

$-1120$

$-1420$

$-553$

7.829

0.267
S30adi

$-1.507$

0.143

0.193

$-1.393$

0.100

S40iso

$-1.823$

0.164

0.128

$-1.448$

0.035

113

85

218

$-916$

$-402$

$-1012$

$-1331$

$-550$

7.840

0.286

0.267

S40adi
-1.689
0.159
0.159
-1.393

S50iso

S50adi

$-1.764$

0.173

0.098

$-1.342$

0.076

0.092

132

126

82

213

$\begin{array}{lll}82 & 85 & 82 \\ 210 & 223 & 213\end{array}$



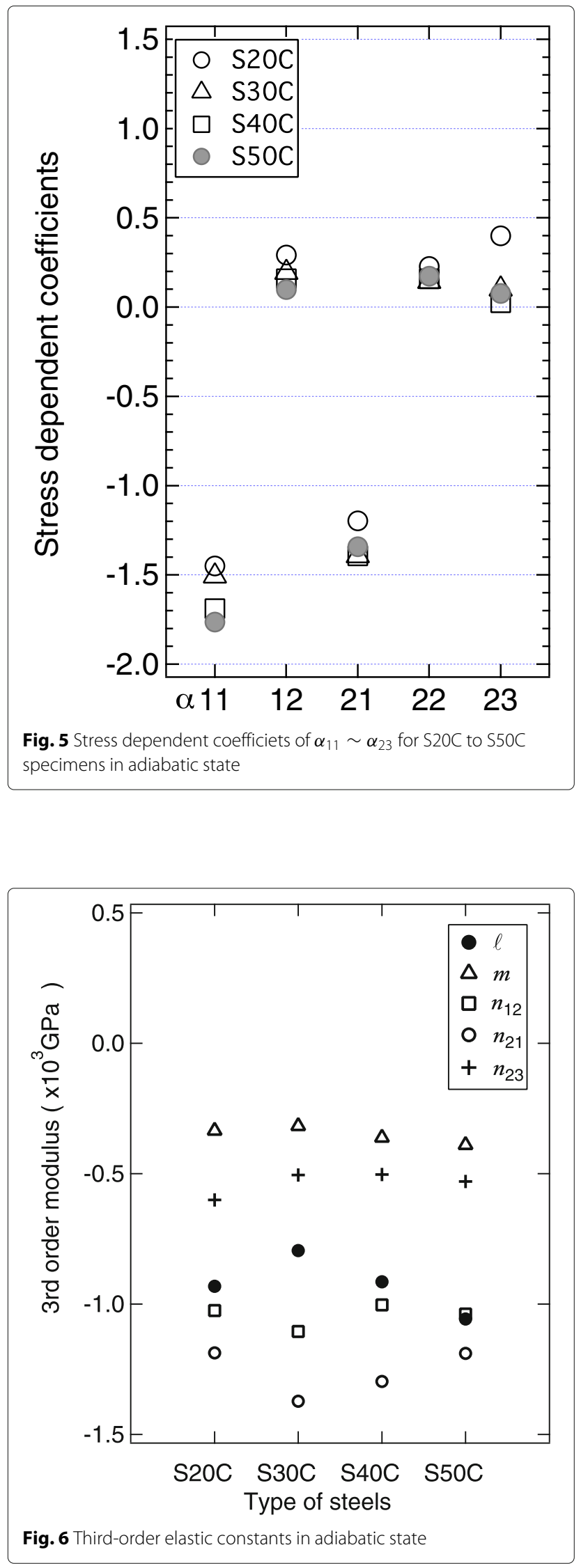

\section{Conclusions}

1. An experimental methods for measuring third-order elastic constants accurately and relatively easy was described.

2. Measurements of the change ratio of propagation time of the elastic wave with respect to the change in the applied stress, stress-strain curves, were performed for four types of practical steel specimens.

3. A formula relating the experimentally measured values to theory was proposed. This formula consisted of stress, Young's modulus, the propagation velocity of the elastic wave, stress dependent coefficient $\alpha_{i j}$, the values of all of which were measurable.

4. The two types of speimens with identical grip sizes but different gauge lengths were prepared to eliminate the influence of the grip parts and to apply uniform stress.

5. A formula for $\alpha_{i j}$ was proposed using data obtained from long and short specimens.

6. $\alpha_{i j}$ was obtained from the gradient of the relationship between the change ratio of the propagation time and stress.

7. $\alpha_{i j}$ is not only a coefficient necessary to obtain the third-order elastic constants but also provide other information on the behavior of elastic wave propagation. The values of $\alpha_{11}$ and $\alpha_{21}$ where the frequency direction of the elastic wave matched the stress axis, were negative and larger in magnitude than those of the other coefficients. This meant a deceleration of the propagation of the elastic wave.

8. The Murnaghan's third-order elastic constants $\ell, m, n$ were obtained for the four types of practical steels under isothermal and adiabatic conditions and those were negative values.

9. The differences between the values of Lamè constants $\lambda, \mu$ and the Young's modulus E measured under isothermal and adiabatic conditions were not very large, but a relatively large difference was found in the case of $m$ and $n$ in third-order modulus.

10. The present study of the third-order elastic constants of materials will greatly contribute to understanding internal stress, thermal properties, Grüneisen constants and so on of steel, semiconductor and non ferrous metals. The author got U.S.Patent on the stress measurement and its equipment (Takahashi 2007).

\section{Abbreviations}

AISI: American iron and steel institute

\section{Acknowledgements}

Not applicable.

Funding

Not applicable. 
Availability of data and materials

Data and materials are available.

\section{Authors' contributions}

The author read and approved the final manuscript.

\section{Authors' information}

$\mathrm{ST}$ is PhD.

\section{Ethics approval and consent to participate}

Not applicable.

\section{Consent for publication}

Author agrees to publication.

\section{Competing interests}

Not applicable.

\section{Publisher's Note}

Springer Nature remains neutral with regard to jurisdictional claims in published maps and institutional affiliations.

Received: 6 August 2017 Accepted: 19 January 2018

Published online: 09 February 2018

\section{References}

Bateman T, Mason WP, McSkimin HJ (1961) Third-order elastic moduli of Germanium. J Appl Phys 32(5):928-936

Egle DM, Bray DE (1976) Measurement of acoustoelastic and third-order elastic constants for rail steel. J Acoust Soc Am 60(3):741-744

Hughes DS, Kelly JL (1953) Second order elastic deformation of solid. Phys Rev 92(5):1145-1149

Krasilnikov OM (1977) Temperature dependence of third-order elastic constants. Sov Phgs State 19(5):764-768

Lazarus D (1949) The variation of the adiabatic elastic constants of $\mathrm{KCl}, \mathrm{NaCl}$, CuZn, Cu and A/ with pressure to 10,000 bars. Phys Rev 76(4):545-553 Murnagham FD (1951) Finite deformation of an elastic solid. Wiley, New York

Takahashi S (2007) Stress measurement method and its apparatus. U.S. Patent No. 7299138, December 10

Takahashi, S, Motegi R (1987) Stress dependency on ultrasonic wave propagation velocity. J Mater Sci 22:1857-1863

Takahashi S, Motegi R (2015) Measurement of third-order elastic constants and applications to loaded structural materals. Springer Plus July 4:325

Takahashi S, Takahashi K (2007) Third order elastic constants of semi continuous casting ingot A3004 aluminium alloy and measurement of stress. J Mater Sci 42:2070-2075

Thurston RN, Brugger K (1964) Third-order elastic constants and the velocity of small amplitude elastic waves in homogeneously stressed media. A Phys Rev 133(6):A1604-A1612

\section{Submit your manuscript to a SpringerOpen ${ }^{\circ}$ journal and benefit from:}

- Convenient online submission

- Rigorous peer review

- Open access: articles freely available online

- High visibility within the field

- Retaining the copyright to your article

Submit your next manuscript at $\gg$ springeropen.com 